\title{
Poincaré invariance of a quantized duality symmetric theory
}

\author{
H. O. Girotti \\ Instituto de Física, Universidade Federal do Rio Grande do Sul, Caixa Postal 15051, 91501-970 - Porto Alegre, RS, Brazil \\ (Received 15 October 1996)
}

\begin{abstract}
The noncovariant duality symmetric action put forward by Schwarz and Sen is quantized by means of the Dirac brackets quantization procedure. The resulting quantum theory is shown to be, nevertheless, relativistically invariant. [S0556-2821(97)01106-5]
\end{abstract}

PACS number(s): 11.30.Cp, 11.25.Mj, 11.30.Ly invariant actions which are also invariant under discrete duality transformations. In particular, the duality symmetric generalization of the four-dimensional Maxwell action involves two gauge potentials $A^{\mu, a}(0 \leqslant \mu \leqslant 3,1 \leqslant a \leqslant 2)$ and reads [2]

$$
S=-\frac{1}{2} \int d^{4} x\left(B^{a, i} \epsilon_{a b} E^{b, i}+B^{a, i} B^{a, i}\right),
$$

where

$$
\begin{gathered}
E^{a, i}=-F^{a, 0 i}=-\left(\partial^{0} A^{a, i}-\partial^{i} A^{a, 0}\right), \\
B^{a, i}=-\frac{1}{2} \epsilon^{i j k} F_{j k}^{a}=-\epsilon^{i j k} \partial_{j} A_{k}^{a},
\end{gathered}
$$

and $1 \leqslant i, j, k \leqslant 3 . S$ is separately invariant under the local gauge transformations

$$
\begin{gathered}
A^{a, 0} \rightarrow A^{a, 0}+\Psi^{a}, \\
A^{a, i} \rightarrow A^{a, i}-\partial^{i} \Lambda^{a},
\end{gathered}
$$

and under the discrete duality transformations

$$
A^{a, \mu} \rightarrow \epsilon_{a b} A^{b, \mu}
$$

The use of the equations of motion

$$
\boldsymbol{\epsilon}^{i j k} \epsilon_{a b} \partial_{0} \partial_{j} A_{k}^{b}+\partial_{j}\left(\partial^{j} A^{a, i}-\partial^{i} A^{a, j}\right)=0
$$

allows for the elimination from $S$ of one of the gauge fields, the action for the remaining one being the conventional Maxwell action.

In terms of the gauge potentials, the Lagrangian density in Eq. (1) reads

$$
\begin{aligned}
\mathcal{L}= & \frac{1}{2} \epsilon^{j k i}\left(\partial_{j} A_{k}^{a}\right) \epsilon_{a b}\left(\partial_{0} A_{i}^{b}\right)-\frac{1}{2} \epsilon^{j k i}\left(\partial_{j} A_{k}^{a}\right) \epsilon_{a b}\left(\partial_{i} A_{0}^{b}\right) \\
& -\frac{1}{4} F^{a, j k} F_{j k}^{a} .
\end{aligned}
$$

Clearly, $\mathcal{L}$ is not a Lorentz scalar. Some alternatives have been suggested to reconcile, already at the classical level, duality and Lorentz symmetries $[3,4]$. In this paper we demonstrate that the quantum field theory arising from Eq. (6) is, nevertheless, relativistically invariant.
In Ref. [1], Schwarz and Sen proposed a class of gauge-

The present work can be summarized as follows. We start by presenting the Hamiltonian formulation of the model before gauge fixing. After choosing the Coulomb gauge, the theory is quantized by means of the Dirac brackets quantization procedure [5-8]. The resulting quantum theory turns out to be local and quantum mechanically consistent. The next step consists in building a set of composite operators which will be shown to verify the Dirac-Schwinger algebra $[9,10]$. As a consequence, a set of charges obeying the Poincare algebra exist and can inmediately be constructed. We prove, afterwards, that the full set of composite operators obeying the Dirac-Schwinger algebra are the components of a second-rank symmetric tensor. The transformation properties of the basic fields under the Poincare group are also studied and serve to demonstrate that the noncovariant Coulomb gauge condition is preserved under Lorentz boosts. We end by arguing that our results can be generalized for an arbitrary canonical gauge.

The canonical Hamiltonian $\left(H_{c}\right)$ following from Eq. (6) reads

$$
H_{c}=\int d^{3} x\left[\frac{1}{2} \epsilon^{j k i}\left(\partial_{j} A_{k}^{a}\right) \epsilon_{a b}\left(\partial_{i} A_{0}^{b}\right)+\frac{1}{4} F^{a, j k} F_{j k}^{a}\right] .
$$

Furthermore, the system possesses the primary constraints

$$
\begin{gathered}
\Omega_{0}^{a} \equiv \pi_{0}^{a} \approx 0, \\
\Omega_{i}^{a} \equiv \pi_{i}^{a}+\frac{1}{2} \epsilon_{a b} \epsilon_{i j k} \partial^{j} A^{b, k} \approx 0,
\end{gathered}
$$

where we have designated by $\pi_{\mu}^{a}$ the momentum canonically conjugate to $A^{a, \mu}$. Then, the total Hamiltonian $\left(H^{\prime}\right)$ is given by $H^{\prime}=H_{c}+\int d^{3} x\left(u^{a, 0} \Omega_{0}^{a}+u^{a, i} \Omega_{i}^{a}\right)$, where the $u$ 's are Lagrange multipliers. Persistence in time of $\Omega_{0}^{a}$ produces neither secondary constraints nor determines the Lagrange multipliers. On the other hand, persistence in time of the primary constraints $\left\{\Omega_{i}^{a}\right\}$ does not lead to the existence of secondary constraints but determines partially the Lagrange multipliers $\left\{u_{i}^{a}\right\}$. Indeed, since the Poissons brackets [12]

$$
\left[\Omega_{i}^{a}(\vec{x}), \Omega_{j}^{b}(\vec{y})\right]_{P}=-\epsilon_{a b} \epsilon_{i j k} \partial_{x}^{j} \delta(\vec{x}-\vec{y})
$$

do not vanish, $\dot{\Omega}_{i}^{a}=\left[\Omega_{i}^{a}, H^{\prime}\right]_{P} \approx 0$ yields $u^{a, i}=$ $\epsilon_{a b}\left(B^{b, i}-\partial^{i} \phi^{b}\right)$, where $\phi^{a}$ is an arbitrary scalar. Thus, 


$$
\Omega^{a}(\vec{x})=\partial^{i} \Omega_{i}^{a}(\vec{x}) \approx 0
$$

and $\Omega_{0}^{a} \approx 0$ are the first-class constraints in the theory [11].

To isolate the second-class constraints from Eq. (8b), we split $\Omega_{i}^{a}$ into longitudinal $(L)$ and transversal $(T)$ components, namely, $\quad \Omega_{i}^{a}=\Omega_{L i}^{a}+\Omega_{T i}^{a} \quad$ where $\quad \Omega_{L i}^{a}$ $=-\left(\partial_{i} \partial^{j} / \nabla^{2}\right) \Omega_{j}^{a}, \quad \Omega_{T i}^{a}=\left(g_{i}^{j}+\partial_{i} \partial^{j} / \nabla^{2}\right) \Omega_{j}^{a}, \quad$ and $\nabla^{2} \equiv-\partial_{j} \partial^{j}$. The first-class constraint (10) only involves the longitudinal components $\Omega_{L i}^{a}$ and states that these components vanish individually. Then, the second-class constraints are

$$
\Omega_{T i}^{a}=\pi_{T i}^{a}+\frac{1}{2} \epsilon_{a b} \epsilon_{i j k} \partial^{j} A_{T}^{b, k} \approx 0
$$

The determination of the constraint structure is over. It only remains to be mentioned that the gauge potential $A^{a, \mu}$, when acted upon by the generator of infinitesimal gauge transformations, $G=\int d^{3} x\left(\Psi^{a} \Omega_{0}^{a}+\Lambda^{a} \Omega^{a}\right)$, undergoes the change $A^{a, \mu} \rightarrow A^{a, \mu}+\delta A^{a, \mu}$ with $\delta A^{a, 0}=\left[A^{a, 0}, G\right]_{P}=\Psi^{a}$ and $\delta A^{a, i}=\left[A^{a, i}, G\right]_{P}=-\partial^{i} \Lambda^{a}$, in agreement with Eq. (3).

We shall next quantize the model by means of the Dirac brackets quantization procedure [5-8]. To this end, we start by fixing the gauge through the subsidiary conditions

$$
\begin{aligned}
& \chi^{a, 0} \equiv A^{a, 0} \approx 0, \\
& \chi^{a} \equiv \partial_{i} A^{a, i} \approx 0 .
\end{aligned}
$$

The formulation of the quantum dynamics of a gauge theory in the Coulomb (physical) gauge is of importance for understanding its structural aspects. The fact that the Coulomb condition and $A^{a, 0} \approx 0$ are, when acting together, accessible gauge conditions is a peculiarity of the model under analysis. This is not the case, for instance, in quantum electrodynamics.

Since the full set of constraints and gauge conditions is, by construction, second class, Dirac brackets with respect to them can be introduced in the usual manner. Afterwards, the phase-space variables are promoted to operators obeying an equal-time commutator algebra which is to be abstracted from the corresponding Dirac brackets algebra, the constraints and gauge conditions thereby translating into strong operator relations. This is the Dirac bracket quantization procedure, which presently yields $[13,14]$

$$
\begin{gathered}
{\left[A_{T}^{a, i}(\vec{x}), A_{T}^{b, j}(\vec{y})\right]=-i \epsilon_{a b} \epsilon^{i j k} \frac{\partial_{k}^{x}}{\nabla^{2}} \delta(\vec{x}-\vec{y}),} \\
{\left[A_{T}^{a, i}(\vec{x}), \pi_{T j}^{b}(\vec{y})\right]=\frac{i}{2} \delta_{a b}\left(g_{j}^{i}+\frac{\partial_{x}^{i} \partial_{j}^{x}}{\nabla^{2}}\right) \delta(\vec{x}-\vec{y}),} \\
{\left[\pi_{T i}^{a}(\vec{x}), \pi_{T j}^{b}(\vec{y})\right]=\frac{i}{4} \epsilon_{a b} \epsilon_{i j k} \partial_{x}^{k} \delta(\vec{x}-\vec{y}) .}
\end{gathered}
$$

As for the quantum mechanical Hamiltonian $(H)$, it can be read off from Eq. (7) after taking into account that constraints and gauge conditions act, within the algebra (13), as strong operator identities. Then

$$
H=\frac{1}{4} \int d^{3} x F^{a, j k} F_{j k}^{a}=-\frac{1}{2} \int d^{3} x B^{a, j} B_{j}^{a} .
$$

One may wonder whether the right-hand side of Eq. (14) is afflicted by ordering ambiguities. However, this not so, since

$$
\left[B^{a, i}(\vec{x}), B^{b, j}(\vec{y})\right]=i \epsilon_{a b} \epsilon^{i j k} \partial_{k}^{x} \delta(\vec{x}-\vec{y})
$$

as follows from Eqs. (13a) and (2b).

The Hamilton equations of motion arising from Eqs. (13) and (14) are

$$
\begin{aligned}
& \mathcal{D}_{i k}^{(-) a b} A_{T}^{b, k}=0, \\
& \partial_{0} \pi_{T i}^{a}=\frac{1}{2} \partial^{j} F_{j i}^{a},
\end{aligned}
$$

where

$$
\mathcal{D}_{i k}^{( \pm) a b} \equiv g_{i k} \delta_{a b} \partial_{0} \pm \epsilon_{a b} \epsilon_{i j k} \partial^{j}
$$

Notice that, in the Coulomb gauge, the Lagrange equation of motion (5) can be cast as

$$
\epsilon^{j l i} \partial_{l} \mathcal{D}_{i k}^{(-) a b} A_{T}^{b, k}=0 \Rightarrow \mathcal{D}_{i k}^{(-) a b} A_{T}^{b, k}=\partial_{i} \xi^{a} .
$$

Since $\partial^{i} \mathcal{D}_{i k}^{(-) a b} A_{T}^{b, k}=0$, the function $\xi^{a}$ must verify $\nabla^{2} \xi^{a}=0$ but is otherwise arbitrary. Thus, the Lagrangian and the Hamiltonian formulations lead to equivalent equations of motions only after the introduction of a regularity requirement at spatial infinity. This situation resembles that encountered in connection with the theory of the two-dimensional $\left[x^{0}, x^{1}, x^{ \pm}=1 / \sqrt{2}\left(x^{0} \pm x^{1}\right)\right]$ self-dual field $(\Phi)$ put forward by Floreanini and Jackiw $[15,16]$, where the equations of motion in the Lagrangian and Hamiltonian formulations turn out to be, respectively, $\partial_{1} \partial_{-} \Phi=0$ and $\partial_{-} \Phi=0$. We also recall that in order to solve $\partial_{-} \Phi=0$ one starts by realizing that $\partial_{-} \Phi=0 \Rightarrow \partial_{+} \partial_{-} \Phi=0 \Rightarrow \square \Phi=0$. The solutions of $\partial_{-} \Phi=0$ are then contained in the field of solutions of $\square \Phi=0$. We shall follow here a similar approach, since

$$
\mathcal{D}_{i k}^{(-) a b} A_{T}^{b, k}=0 \Rightarrow \mathcal{D}^{(+) c a, l i} \mathcal{D}_{i k}^{(-) a b} A_{T}^{b, k}=0 \Rightarrow \square A_{T}^{c, l}=0 .
$$

The solving of $\square A_{T}^{a, i}=0$ leads to

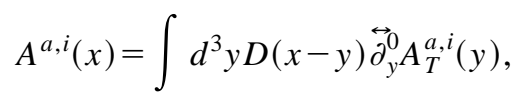

where $D(x-y)$ is the zero-mass Pauli-Jordan $\delta$ function and $\left(A \stackrel{\leftrightarrow}{\partial^{k}} B\right) \equiv A \partial^{k} B-B \partial^{k} A$. From this last equation and Eq. (13) follows that the field commutator at different space-time points reads

$$
\begin{aligned}
& {\left[A_{T}^{a, i}(x), A_{T}^{b, j}(y)\right]} \\
& \quad=i\left[\delta_{a b}\left(g^{i j}+\frac{\partial_{x}^{i} \partial_{x}^{j}}{\nabla_{x}^{2}}\right)-\epsilon_{a b} \epsilon^{i j k} \frac{\partial_{k}^{x} \partial_{0}^{x}}{\nabla_{x}^{2}}\right] D(x-y) .
\end{aligned}
$$

By applying $\mathcal{D}_{k i}^{(-) c a}(x)$ to both sides of Eq. (21), one can check that the field configurations entering the just men- 
tioned commutator are in fact solutions of (16a). As known, the function $D(x-y)$ can be given as the sum of a positive plus a negative frequency part and we, therefore, can write

$$
A_{T}^{a, i}(x)=A_{T}^{a, i(+)}(x)+A_{T}^{a, i(-)}(x),
$$

where

$$
\begin{aligned}
A_{T}^{a, i( \pm)}(x)= & \frac{1}{(2 \pi)^{3 / 2}} \int \frac{d^{3} k}{\sqrt{2|\vec{k}|}} \exp \left[ \pm i\left(|\vec{k}| x^{0}-\vec{k} \cdot \vec{x}\right)\right] \\
& \times \sum_{\lambda=1}^{2} \varepsilon_{\lambda}^{a, i}(\vec{k}) a_{\lambda}^{( \pm)}(\vec{k})
\end{aligned}
$$

and $\varepsilon_{\lambda}^{a, i}(\vec{k}), \lambda=1,2$, are unit norm polarization vectors. By going back with Eq. (23) into Eq. (21) one obtains

$$
\begin{aligned}
& \sum_{\lambda, \lambda^{\prime}=1}^{2} \varepsilon_{\lambda}^{a, i}(\vec{k}) \varepsilon_{\lambda^{\prime}}^{b, j}\left(\overrightarrow{k^{\prime}}\right)\left[a_{\lambda}^{(-)}(\vec{k}), a_{\lambda^{\prime}}^{(+)}\left(\overrightarrow{k^{\prime}}\right)\right] \\
& \quad=\left[-\delta_{a b}\left(g^{i j}+\frac{k^{i} k^{j}}{|\vec{k}|}\right)+\epsilon_{a b} \epsilon^{i j l} \frac{k_{l}}{|\vec{k}|}\right] \delta\left(\vec{k}-\overrightarrow{k^{\prime}}\right),
\end{aligned}
$$

while all others commutators vanish. The polarization vectors are to be found by replacing (23) into the gauge condition (12b) and the equation of motion (16a). In this way we arrive, respectively, at $k_{i} \varepsilon_{\lambda}^{a, i}(\vec{k})=0$ and

$$
\sum_{i j}^{a b}(\vec{k}) \varepsilon_{\lambda}^{b, j}=0
$$

where

$$
\Sigma_{i j}^{a b}(\vec{k}) \equiv g_{i j} \delta_{a b} k_{0}-\epsilon_{a b} \epsilon_{i l j} k^{l}
$$

The vanishing of the determinant of the matrix $\Sigma_{i j}^{a b}$ is a necessary and sufficient condition for the homogeneous system of equations in Eq. (25) to have a solution different from the trivial one $\varepsilon_{\lambda}^{b, j}=0$. In the present case this determinant is proportional to $k^{2}$ and its vanishing merely states that the theory only propagates zero-mass particles. Furthermore, Eq. (25) also implies that $\varepsilon_{\lambda}^{a, i} \sum_{i j}^{a b} \varepsilon_{\lambda}^{b, j}=0$. This nontrivial relationship among the polarization vectors associated with different gauge potentials can be cast as

$$
\sum_{\lambda=1}^{2} \vec{\varepsilon}_{\lambda}^{a}(\vec{k}) \times \vec{\varepsilon}_{\lambda}^{b}(\vec{k})=-2 \epsilon_{a b} \frac{\vec{k}}{|\vec{k}|} .
$$

On the other hand, the Coulomb gauge polarization vectors span, by construction, the space orthogonal to $\vec{k}$ : i.e.,

$$
\sum_{\lambda=1}^{2} \varepsilon_{\lambda}^{a, i}(\vec{k}) \varepsilon_{\lambda}^{a, j}(\vec{k})=-\left(g^{i j}+\frac{k^{i} k^{j}}{|\vec{k}|^{2}}\right) .
$$

By using Eqs. (27) and (28) we can solve at once for the commutator in Eq. (24) and find

$$
\left[a_{\lambda}^{(-)}(\vec{k}), a_{\lambda^{\prime}}^{(+)}\left(\overrightarrow{k^{\prime}}\right)\right]=\delta_{\lambda \lambda^{\prime}} \delta\left(\vec{k}-\vec{k}^{\prime}\right) .
$$

Thus the space of states is, as expected, a Fock space with positive definite metric.
Hence, the quantization of the Schwarz-Sen model has led to a local and physically sensible quantum field theory. Our next task is to demonstrate that this quantum theory is also relativistically invariant.

We are therefore looking for a set of composite operators $\left\{\Theta_{\mu \nu}\right\}$ which may serve as Poincaré densities. By experience, we try to build them according to the rules

$$
\Theta_{\mu \nu}=T_{\mu \nu}+\partial^{\lambda} \psi_{\underline{\lambda \mu \nu}},
$$

where

$$
\begin{aligned}
& T_{\mu \nu}=\frac{\partial \mathcal{L}}{\partial\left(\partial^{\mu} A^{a, \rho}\right)} \partial_{\nu} A^{a, \rho}-g_{\mu \nu} \mathcal{L}, \\
& \psi_{\underline{\lambda \mu \nu}}=\frac{1}{2}\left(S_{\underline{\lambda \mu \nu}}+S_{\underline{\nu \mu \lambda}}+S_{\underline{\lambda \nu} \mu}\right), \\
& S_{\underline{\lambda \mu \nu}}=-\frac{\partial \mathcal{L}}{\partial\left(\partial^{\nu} A^{a, \alpha}\right)} \mathcal{A}_{\underline{\lambda \mu} \beta}^{\alpha} A^{a, \beta},
\end{aligned}
$$

and $\mathcal{A}_{\lambda \mu \beta}^{\alpha}=g_{\lambda}^{\alpha} g_{\mu \beta}-g_{\mu}^{\alpha} g_{\lambda \beta}$. Clearly, $\psi, S$, and $\mathcal{A}$ are antisymmetric under the exchange of the underlined indices. These are, of course, the standard rules for constructing the symmetric (Belinfante) energy-momentum tensor. However, we cannot yet decide on whether or not $\Theta$ is a tensor [14]. By bringing Eq. (6) into Eqs. (31) and (33) one obtains

$$
\Theta_{00}=-\frac{1}{2} B^{a, i} B_{i}^{a}
$$

$$
\begin{aligned}
& \Theta_{0 i}=\Theta_{i 0}=-\frac{1}{2} \epsilon_{i j k} \epsilon_{a b} B^{a, j} B^{b, k}, \\
& \Theta_{i j}=\Theta_{j i}=-B_{i}^{a} B_{j}^{a}+g_{i j} B^{a, l} B_{l}^{a} .
\end{aligned}
$$

Thus, $\Theta$ is symmetric and free of ordering ambiguities.

We look next for the equal-time commutator algebra obeyed by the components of $\Theta$. According to Eq. (34), this algebra is fully determined by the commutator (15). In particular, one can corroborate that

$$
\begin{aligned}
& {\left[\Theta^{00}\left(x^{0}, \vec{x}\right), \Theta^{00}\left(x^{0}, \vec{y}\right)\right]} \\
& \quad=-i\left\{\Theta^{0 k}\left(x^{0}, \vec{x}\right)+\Theta^{0 k}\left(x^{0}, \vec{y}\right)\right\} \partial_{k}^{x} \delta(\vec{x}-\vec{y}),
\end{aligned}
$$

$$
\begin{aligned}
& {\left[\Theta^{00}\left(x^{0}, \vec{x}\right), \Theta^{0 k}\left(x^{0}, \vec{y}\right)\right]} \\
& \quad=-i\left\{\Theta^{k j}\left(x^{0}, \vec{x}\right)-g^{k j} \Theta^{00}\left(x^{0}, \vec{y}\right)\right\} \partial_{j}^{x} \delta(\vec{x}-\vec{y}),
\end{aligned}
$$

$$
\begin{aligned}
& {\left[\Theta^{0 k}\left(x^{0}, \vec{x}\right), \Theta^{0 j}\left(x^{0}, \vec{y}\right)\right]} \\
& \quad=i\left\{\Theta^{0 k}\left(x^{0}, \vec{y}\right) \partial_{x}^{j}+\Theta^{0 j}\left(x^{0}, \vec{x}\right) \partial_{x}^{k}\right\} \delta(\vec{x}-\vec{y}),
\end{aligned}
$$

which is just the Dirac-Schwinger algebra [9]. As is well known [9], this guarantees that the charges 


$$
\begin{gathered}
P^{\mu} \equiv \int d^{3} x \Theta^{0 \mu}, \\
J^{\mu \nu} \equiv \int d^{3} x\left(\Theta^{0 \mu} x^{\nu}-\Theta^{0 \nu} x^{\mu}\right),
\end{gathered}
$$

obey the Poincaré algebra, i.e., $\left[P^{\mu}, P^{\nu}\right]=0$, $\left[J^{\mu \nu}, P^{\sigma}\right]=i\left(g^{\mu \sigma} P^{\nu}-g^{\nu \sigma} P^{\mu}\right)$, and $\left[J^{\mu \nu}, J^{\rho \sigma}\right]=i\left(g^{\mu \rho} J^{\nu \sigma}\right.$ $\left.+g^{\nu \sigma} J^{\mu \rho}-g^{\mu \sigma} J^{\nu \rho}-g^{\nu \rho} J^{\mu \sigma}\right)$.

It takes just a few more steps to demonstrate that $\Theta$ is a tensor. Indeed, the additional equal-time commutators $\left[\Theta^{i j}\left(x^{0}, \vec{x}\right), \Theta^{00}\left(x^{0}, \vec{y}\right)\right]$ and $\left[\Theta^{i j}\left(x^{0}, \vec{x}\right), \Theta^{0 k}\left(x^{0}, \vec{y}\right)\right]$ can also be readily evaluated by using Eqs. (34) and (15). These results and Eq. (35) can be collected into

$$
\begin{gathered}
{\left[P^{\mu}, \Theta^{\alpha \beta}\right]=-i \partial^{\mu} \Theta^{\alpha \beta},} \\
{\left[J^{\mu \nu}, \Theta^{\alpha \beta}\right]=-i\left(x^{\nu} \partial^{\mu}-x^{\mu} \partial^{\nu}\right) \Theta^{\alpha \beta}-i\left(\Theta^{\mu \alpha} g^{\nu \beta}+\Theta^{\mu \beta} g^{\nu \alpha}\right.} \\
\left.-\Theta^{\nu \alpha} g^{\mu \beta}-\Theta^{\nu \beta} g^{\mu \alpha}\right),
\end{gathered}
$$

which are, respectively, the translation and rotation transformation laws to be obeyed by a second-rank tensor [17]. The purported proof of relativistic invariance of the quantized Schwarz-Sen theory is now complete.

What remains to be done is to demonstrate that the Coulomb gauge formulation of the quantized Schwarz-Sen theory is in fact covariant. Since translations and ordinary rotations do not destroy the Coulomb gauge condition we concentrate on Lorentz boosts. By using (36b), (34), (2b), and (13a) one finds that

$$
-i\left[J^{0 k}, A_{T}^{a, i}\right]=\left(x^{0} \partial^{k}-x^{k} \partial^{0}\right) A_{T}^{a, i}-\epsilon_{a b} \epsilon^{k l j} \frac{\partial^{i} \partial_{l}}{\nabla^{2}} A_{T j}^{b}
$$

The term proportional to $\epsilon_{a b}$ signalizes that gauge potentials corresponding to different values of $a$ get mixed by Lorentz boosts. This does not occur for ordinary rotations. Furthermore, the mixing term in Eq. (38) describes an operator gauge transformation, which, as one easily verifies, makes this commutator compatible with the transversality condition $\partial_{i} A_{T}^{a, i}=0$. Hence, under Lorentz boosts, the field $A_{T}^{a, i}$ undergoes, besides the usual vector transformation, an operator gauge transformation which restores the Coulomb gauge in the new Lorentz frame [18].

Although this work has been entirely carried out within the Coulomb gauge, we observe that the quantized SchwarzSen model turned out to be a local theory fully formulated in terms of the gauge invariant fields $A_{T}^{a, j}, a=1,2$. Therefore, our conclusions about relativistic invariance apply equally well for all canonical gauges.

This work was supported in part by Conselho Nacional de Desenvolvimento Científico e Tecnológico (CNPq), Brazil.
[1] J. H. Schwarz and A. Sen, Nucl. Phys. B411, 35 (1994).

[2] Our space-time metric is $g_{00}=-g_{11}=-g_{22}=1$ while $\epsilon_{a b}$ designates a generic element of the two-dimensional antisymmetric unit matrix $\left(\epsilon_{12}=+1\right)$.

[3] A. Khoudeir and N. Pantoja, Phys. Rev. D 53, 5974 (1996).

[4] P. Pasti, D. Sorokin, and M. Tonin, Phys. Lett. B 352, 59 (1995); Phys. Rev. D 52, R4277 (1995).

[5] P. A. M. Dirac, Lectures on Quantum Mechanics (Belfer Graduate School on Science, Yeshiva University, New York, 1964).

[6] E. S. Fradkin and G. A. Vilkovisky, CERN Report No. TH 2332, 1977 (unpublished).

[7] K. Sundermeyer, Constrained Dynamics (Springer-Verlag, Berlin, 1982).

[8] H. O. Girotti, Classical and Quantum Dynamics of Constrained Systems, Lectures in Proceedings of the $\mathrm{V}^{\text {th }}$ Jorge Andre Swieca Summer School, edited by O. J. P. Eboli, M. Gomes, and A. Santoro (World Scientific, Singapore, 1990), pp. $1-77$.

[9] J. Schwinger, Phys. Rev. 127, 324 (1962). Also see C. Itzyk- son and J-B. Zuber, Quantum Field Theory (McGraw-Hill, Singapore, 1985).

[10] J. Schwinger, Particles, Sources and Fields (Addison-Wesley, New York, 1970), Vol. I.

[11] I. Martin and A. Restuccia, Phys. Lett. B 323, 311 (1994).

[12] The time label in the arguments of the fields will be omitted whenever it does not lead to confusion.

[13] We shall not distinguish between a quantum field operator and its classical counterpart.

[14] Recall that $\mathcal{L}$ is not Lorentz scalar and that a set of four quantities $A^{a, \mu}, \mu=0,1,2,3$, obeying the Coulomb condition do not transform as components of a four-vector under Lorentz boosts.

[15] R. Floreanini and R. Jackiw, Phys. Rev. Lett. 59, 1873 (1987).

[16] M. E. V. Costa and H. O. Girotti, Phys. Rev. Lett. 60, 1771 (1988); F. P. Devecchi and H. O. Girotti, Phys. Rev. D 49, 4302 (1994).

[17] J. Schwinger, Phys. Rev. 130, 406 (1963).

[18] B. Zumino, J. Math. Phys. (N.Y.) 1, 1 (1960). 